\title{
Second derivatives of norms and contractive complementation in vector-valued spaces
}

\author{
by \\ Bas Lemmens (Coventry), Beata Randrianantoanina (Oxford, OH) \\ and ONNO VAN GaAns (Leiden)
}

\begin{abstract}
We consider 1-complemented subspaces (ranges of contractive projections) of vector-valued spaces $\ell_{p}(X)$, where $X$ is a Banach space with a 1-unconditional basis and $p \in(1,2) \cup(2, \infty)$. If the norm of $X$ is twice continuously differentiable and satisfies certain conditions connecting the norm and the notion of disjointness with respect to the basis, then we prove that every 1-complemented subspace of $\ell_{p}(X)$ admits a basis of mutually disjoint elements. Moreover, we show that every contractive projection is then an averaging operator. We apply our results to the space $\ell_{p}\left(\ell_{q}\right)$ with $p, q \in(1,2) \cup(2, \infty)$ and obtain a complete characterization of its 1-complemented subspaces.
\end{abstract}

1. Introduction. It is well known that every orthogonal projection on a Hilbert space $H$ is contractive and that for every closed subspace $Y$ of $H$ there exists an orthogonal projection whose range is $Y$. In fact, if the dimension of $H$ is at least 3, then it has been shown by Kakutani [12] that this property characterizes the Hilbert spaces among the Banach spaces. Hence contractive projections on Banach spaces are a natural generalization of orthogonal projections on Hilbert spaces; but, as the result of Kakutani indicates, they are more rare. It is therefore not surprising that contractive projections have been studied extensively, starting with work by Bohnenblust [3] in the nineteen forties. A detailed overview of the vast literature on contractive projections and their applications can be found in the survey papers $[5,20]$.

Identifying contractive projections and their ranges for a given Banach space has often proved to be difficult. For Lebesgue $L_{p}$ spaces, with $p \neq 2$, there exists a well known characterization of the contractive projections and their ranges (see $[1,2,9,18,26]$ ). However, for many other classical Banach

2000 Mathematics Subject Classification: Primary 46B45, 46B04; Secondary 47B37.

Key words and phrases: block basis, contractive projections, $\ell_{p}\left(\ell_{q}\right)$ spaces, norm one projections, one-complemented subspaces, smooth norms, vector-valued spaces.

B.R.: participant, NSF Workshop in Linear Analysis and Probability, Texas A\&M University. 
spaces, such as Orlicz spaces and Lorentz sequence spaces, there are only partial results; see the survey [20]. The best results to date for MusielakOrlicz spaces were obtained by Jamison, Kamińska, and Lewicki [11], who characterized the 1-complemented subspaces of finite co-dimension in case the Orlicz functions are sufficiently smooth.

The problem of characterizing contractive projections and their ranges in vector-valued spaces is known to be particularly hard, even for spaces of the type $L_{p}(X)$ where $X$ is finite-dimensional. We refer the reader to a survey by Doust [10] for a nice overview of the various partial results that were known in the mid-nineties. As Doust points out, most of the results require special additional assumptions on the form of projections and leave open the cases of $L_{p}(X)$ and $\ell_{p}(X)$, even when $X$ is a two-dimensional $\ell_{q}$ space. The most general result for vector-valued spaces was obtained by Raynaud [23], who gave a complete description of the contractive projections in $L_{p}(H)$ for $H$ a Hilbert space. Raynaud's result is valid in full generality without any assumptions about $\sigma$-finiteness of the measure on $L_{p}$ or separability of the Hilbert space $H$.

In the present paper we study contractive projections in vector-valued $\ell_{p}(X)$ spaces, in particular $\ell_{p}\left(\ell_{q}\right)$ spaces. Our main result characterizes the 1 -complemented subspaces of real $\ell_{p}\left(\ell_{q}\right)$ spaces for $p, q \in(1,2) \cup(2, \infty)$. The complex case was obtained in [19], in which 1-complemented subspaces of general complex spaces with 1-unconditional bases are characterized, using a technique based on Hermitian operators. This technique, however, does not work in real spaces. This is due to the following fundamental result of Kalton and Wood [13] for complex spaces, which has no real analogue (see [17]): every 1-complemented subspace of a complex Banach space with a 1-unconditional basis has a 1-unconditional basis. In fact, it is not known whether each 1-complemented subspace in a real space admits an unconditional basis with any constant (cf. [20, Section 7.e]). The characterization presented here shows in particular that every 1-complemented subspace of a real $\ell_{p}\left(\ell_{q}\right)$ space has an unconditional basis.

To obtain the characterization, we introduce a condition on the second derivative of the norm of a real Banach space with a 1-unconditional basis that guarantees that every contractive projection is an averaging operator and its range admits a block basis. We subsequently apply it to analyse contractive projections on vector-valued $\ell_{p}(X)$ spaces. As a consequence we find that 1-complemented subspaces of $\ell_{p}\left(\ell_{q}\right)$ admit a block basis, in case $p$ and $q$ are both in $(2, \infty)$ or both in $(1,2)$. In the mixed case, where $p \in(1,2)$ and $q \in(2, \infty)$ or the other way around, we cannot apply the condition and we shall use a different argument.

The idea to exploit the second derivative of the norm to analyse contractive projections is not new. In fact, it already appears implicitly in work of 
Bernau and Lacey [2] and Lindenstrauss and Tzafriri [18], who considered the derivative of the norming functionals (or duality map). The derivative of the norming functionals has also been used by Bru, Heinich, and Lootgieter [4] to identify contractive projections on Orlicz spaces that have a second order smooth norm and satisfy some additional constraints. More recently, Lemmens and van Gaans [15] have used the second derivative of the norm to show for a fairly general class of finite-dimensional Banach spaces that the range of every contractive projection has a block basis. In particular, one could apply their technique to prove that the range of each contractive projection on $\ell_{p}^{m}\left(\ell_{q}^{n}\right)$, where $m, n \in \mathbb{N}$, has a block basis, if $p>2$ and $q>2$, or $p$ and $q$ are both in $(1,2)$. It is interesting to note that differential techniques are not only useful for analysing contractive projections, but also appear in the study of isometries; see, for instance, work by Koldobskiu [14].

2. Preliminaries. In this section we collect several definitions and notations that will be used throughout the exposition. In addition, we recall some preliminary results. Before we get started, however, we emphasize that in this paper all Banach spaces are over the field of real numbers.

Let $X$ be a Banach space with a 1 -unconditional basis $\left\{e_{i}\right\}_{i=1}^{\infty}$. We denote by $S_{X}=\{x \in X:\|x\|=1\}$ the unit sphere of $X$. The dual space of $X$ is denoted by $X^{*}$ and the conjugate norm is indicated by $\|\cdot\|^{*}$. For each $x=\sum_{i=1}^{\infty} \alpha_{i} e_{i}$ in $X$ we let $s(x)=\left\{i \in \mathbb{N}: \alpha_{i} \neq 0\right\}$ be the support of $x$. If $s(x)$ is finite, we call $x$ simple. Since $X$ has a 1-unconditional basis, the partial ordering induced by the basis makes $X$ a Banach lattice. Hence there exists a natural notion of disjointness in $X$. Elements $x$ and $y$ in $X$ are called disjoint if $|x| \wedge|y|=0$. As $X$ has a 1-unconditional basis, this is equivalent to $s(x) \cap s(y)=\emptyset$. We note that the dual of a Banach lattice is again a Banach lattice and therefore there exists a natural notion of disjointness in $X^{*}$. If $Y$ is a subspace of $X$ and $Y$ has a basis $\left\{y_{i}\right\}_{i=1}^{\operatorname{dim} Y}$ such that $y_{i}$ and $y_{j}$ are disjoint for all $i \neq j$, then $\left\{y_{i}\right\}_{i=1}^{\operatorname{dim} Y}$ is called a block basis for $Y$.

In this paper we are particularly interested in the vector-valued spaces $\ell_{p}(X)$. If $X$ is a Banach space with norm $\sigma$, then for $p \in[1, \infty)$ the vectorvalued space $\ell_{p}(X)$ consists of those $x: \mathbb{N} \rightarrow X$ for which

$$
\left(\sum_{k=1}^{\infty} \sigma(x(k))^{p}\right)^{1 / p}<\infty .
$$

By equipping the space $\ell_{p}(X)$ with the norm

$$
\|x\|_{\ell_{p}(X)}=\left(\sum_{k=1}^{\infty} \sigma(x(k))^{p}\right)^{1 / p} \quad \text { for all } x \in \ell_{p}(X),
$$

it becomes a Banach space. It is not difficult to verify that if $X$ has a 1-unconditional basis $\left\{e_{i}\right\}_{i=1}^{\infty}$, then for any ordering on the elements $(i, j) \in$ 
$\mathbb{N} \times \mathbb{N}$ the functions $e_{i j}: \mathbb{N} \rightarrow X$ given by $e_{i j}(k)=e_{i}$ if $k=j$ and $e_{i j}(k)=0$ otherwise form a 1-unconditional basis for $\ell_{p}(X)$. For elements in $\ell_{p}(X)$ with basis $\left\{e_{i j}\right\}_{(i, j)}$, it is useful to introduce the notion of vector support. For $x \in \ell_{p}(X)$ we define vs $(x)=\{k \in \mathbb{N}: x(k) \neq 0\}$ to be the vector support of $x$. We note that the dual space of $\ell_{p}(X)$ is equal to $\ell_{p^{*}}\left(X^{*}\right)$, where $1 / p+1 / p^{*}=1$, if $X$ is reflexive (see e.g. [7, Chapter IV]).

If $X$ is a Banach space with a 1-unconditional basis and $T: X \rightarrow X$ is a linear operator for which there exist mutually disjoint elements $\left\{u_{i}\right\}_{i \in I}$ in $X$ and mutually disjoint elements $\left\{v_{i}^{*}\right\}_{i \in I}$ in $X^{*}$ such that $v_{i}^{*}\left(u_{j}\right)=0$ for all $i \neq j$ and

$$
T x=\sum_{i \in I} v_{i}^{*}(x) u_{i} \quad \text { for all } x \in X,
$$

then $T$ is called an averaging operator. Obviously, the range of an averaging operator has a block basis.

We also need to recall some definitions concerning higher order derivatives of norms. Let $X$ and $Y$ be Banach spaces and let $L(X, Y)$ be the Banach space of continuous linear operators from $X$ into $Y$ equipped with the usual operator norm. We denote by $B^{k}(X, Y)$ the Banach space of continuous $k$-linear operators $T: X \times \cdots \times X \rightarrow Y$ with the norm $\|T\|=$ $\sup \left\{\left\|T\left(x_{1}, \ldots, x_{k}\right)\right\|:\left\|x_{1}\right\|=\cdots=\left\|x_{k}\right\|=1\right\}$. A mapping $\varphi: U \rightarrow Y$, where $U \subset X$ is open, is called differentiable at $x \in U$ if there exists a linear operator $D \varphi(x)$ in $L(X, Y)$ such that

$$
\lim _{h \rightarrow 0} \frac{\|\varphi(x+h)-\varphi(x)-D \varphi(x) h\|}{\|h\|}=0 .
$$

The linear operator $D \varphi(x)$ is unique and is called the derivative of $\varphi$ at $x$. Higher order derivatives $D^{k} \varphi(x) \in B^{k}(X, Y)$ are defined in the usual inductive manner (see Dieudonné [8]). The mapping $\varphi: U \rightarrow Y$ is said to be $C^{k}$ on $U$ if it is $k$ times differentiable at every point $x \in U$ and $D^{k} \varphi: U \rightarrow B^{k}(X, Y)$ is continuous. We note that if $\varphi: U \rightarrow Y$ is $k$ times differentiable at $x$, then the multi-linear map $D^{k} \varphi(x)$ is symmetric.

If $\varphi: U \rightarrow Y$ is $C^{k}$ on $U$ and the line segment $[x, x+h] \subset U$, then the usual Taylor expansion

$$
\varphi(x+h)=\varphi(x)+\sum_{j=1}^{k} \frac{1}{j !} D^{j} \varphi(x)(h, \ldots, h)+\vartheta_{x}(h),
$$

where

$$
\lim _{\|h\| \rightarrow 0} \frac{\left\|\vartheta_{x}(h)\right\|}{\|h\|^{k}}=0
$$

is valid. We shall also use a weaker notion of differentiability. A map $\varphi: U \rightarrow$ $Y$ is said to be $k$ times directionally differentiable at $x \in U$ if for $1 \leq j \leq k$ 
there exists a continuous symmetric $j$-linear operator $D^{j} \varphi(x) \in B^{j}(X, Y)$ such that for every $h \in X$ and $t \in \mathbb{R}$ with $[x, x+t h] \subset U$ we have

$$
\varphi(x+t h)=\varphi(x)+\sum_{j=1}^{k} \frac{t^{j}}{j !} D^{j} \varphi(x)(h, \ldots, h)+\vartheta_{x}(t h),
$$

where

$$
\lim _{t \rightarrow 0} \frac{\left\|\vartheta_{x}(t h)\right\|}{|t|^{k}}=0 .
$$

One can verify that the operators $D^{j} \varphi(x)$ are unique. Equipped with these notions of differentiability we now recall the following definition from [25].

Definition 2.1. A Banach space $X$ is called $C^{k}$-smooth if the norm $\|\cdot\|$ is $C^{k}$ on $X \backslash\{0\}$. It is said to be $D^{k}$-smooth if the norm is $k$ times directionally differentiable at each $x \in X \backslash\{0\}$.

In the analysis of the vector-valued spaces $\ell_{p}(X)$ we shall use the following smoothness result of Leonard and Sundaresan.

Theorem 2.2 ([16, Theorem 3.3]). If $X$ is a Banach space and $p>k$, then $\ell_{p}(X)$ is $C^{k}$-smooth if and only if $X$ is $C^{k}$-smooth and the $k$ th derivative of the norm of $X$ is uniformly bounded on the unit sphere in $X$.

In particular, it follows from this theorem that the space $\ell_{p}\left(\ell_{q}\right)$ is $C^{2}$ smooth if $p$ and $q$ are in $(2, \infty)$.

3. Second derivatives of norms and contractive projections. We begin this section by introducing a property of the second derivative of the norm. Subsequently we explain how it can be used to analyse contractive projections and their ranges.

Definition 3.1. Suppose $X$ is a Banach space with a basis and for any $x, y \in X$ the function $N=N_{x y}: \mathbb{R} \rightarrow \mathbb{R}$ is given by $N(\alpha)=\|x+\alpha y\|$ for all $\alpha \in \mathbb{R}$. We say that $X$ reflects disjointness if for any $x, y \in X$ with $x \notin \operatorname{span}\{y\}$ the following conditions hold:

(i) the function $N$ is continuously differentiable (and then $N^{\prime \prime}(\alpha)$ exists almost everywhere by convexity);

(ii) if $x$ and $y$ are not disjoint and $N^{\prime}(0)=0$, then $N^{\prime \prime}(\alpha)$ does not converge to 0 as $\alpha \rightarrow 0$ along any subset of $[0,1]$ of full measure;

(iii) if $x$ and $y$ are disjoint and $y$ is simple, then $N^{\prime}(0)=0$ and $N^{\prime \prime}(\alpha)$ converges to 0 as $\alpha \rightarrow 0$ along a subset of $[0,1]$ of full measure.

The idea of this definition is that if $X$ reflects disjointness, then one can test disjointness of elements in $X$ by analysing the second derivative of the norm. Similar ways to test disjointness have been applied by Koldobskiı̌ [14] to identify isometries. The connection with contractive projections 
was found by Randrianantoanina [21], who used the condition to identify contractive projections on certain Orlicz sequence spaces equipped with the Luxemburg norm.

Let us now explain the connection of Definition 3.1 with contractive projections. To do this it is useful to recall the following definition from [22].

Definition 3.2. Let $X$ be a Banach space with a basis and let $T: X \rightarrow$ $X$ be a linear operator. We call $T$ semi-band preserving if for any $x, y \in X$ the elements $T x$ and $T y$ are disjoint whenever $T x$ and $y$ are disjoint.

Semi-band preserving operators on a Banach space with a 1-unconditional basis have a special form as the following theorem indicates.

Theorem 3.3 ([22, Theorem 4.7]). If $X$ is a Banach space and $X$ has a 1-unconditional basis, then a linear operator $T: X \rightarrow X$ is semi-band preserving if and only if $T$ is an averaging operator.

Thus, to show that a contractive projection is an averaging operator and its range has a block basis, it suffices to prove that the projection is semi-band preserving. Doing that involves testing disjointness and this is where the property in Definition 3.1 comes into play. As a matter of fact, we have the following result, which generalizes [21, Theorem 3.2].

TheOREM 3.4. If $X$ is a $D^{2}$-smooth Banach space with a 1-unconditional basis, and $X$ reflects disjointness, then every contractive projection on $X$ is an averaging operator and its range admits a block basis.

The proof of Theorem 3.4 is very similar to that of [21, Theorem 3.2] and uses the following lemma, which is a slight modification of [21, Lemma $3.1]$.

Lemma 3.5 ([21, Lemma 3.1]). Let $\varphi: \mathbb{R} \rightarrow[0, \infty)$ and $\psi: \mathbb{R} \rightarrow[0, \infty)$ be convex functions that are continuously differentiable and assume that $\varphi^{\prime}$ and $\psi^{\prime}$ are absolutely continuous on $[0,1]$. If $\varphi(0)=\psi(0)$ and $\varphi(\alpha) \leq \psi(\alpha)$ for all $\alpha \in \mathbb{R}$, then

(a) $\varphi^{\prime}(0)=\psi^{\prime}(0)$

(b) the set $E=\left\{\alpha: \varphi^{\prime \prime}(\alpha)\right.$ and $\psi^{\prime \prime}(\alpha)$ exist and $\left.\varphi^{\prime \prime}(\alpha) \leq \psi^{\prime \prime}(\alpha)\right\}$ has positive Lebesgue measure in each interval $(0, \delta), \delta>0$;

(c) for every $C>0$ the Lebesgue measure of

$$
\left\{\alpha \in[0,1]: \psi^{\prime \prime}(\alpha) \text { exists and } \psi^{\prime \prime}(\alpha) \leq C\right\}
$$

is strictly smaller than 1 whenever $\varphi^{\prime \prime}(\alpha) \rightarrow \infty$ as $\alpha \rightarrow 0$ along a subset of full measure.

Proof. Parts (a) and (c) are as in [21, Lemma 3.1]. Part (b) is a modification of that lemma. Let $A:=\left\{\alpha>0: \varphi^{\prime}(\alpha)=\psi^{\prime}(\alpha)\right\}$. If inf $A>0$, then there is an $\varepsilon \in(0, \inf A)$ such that $\varphi^{\prime}(\alpha) \neq \psi^{\prime}(\alpha)$ for all $\alpha \in(0, \varepsilon)$. Define 
$h:=\psi-\varphi$. Then $h \geq 0$ and $h^{\prime}(\alpha) \neq 0$ for all $\alpha \in(0, \varepsilon)$. As $h^{\prime}$ is continuous, $h^{\prime}$ is either strictly positive or strictly negative on $(0, \varepsilon)$. As $h(0)=0$ and $h \geq 0$, we have $h^{\prime}(\alpha)>0$ for all $\alpha \in(0, \varepsilon)$. Since $h^{\prime}$ is absolutely continuous, $h^{\prime \prime}$ exists almost everywhere and

$$
h^{\prime}(\alpha)=\int_{0}^{\alpha} h^{\prime \prime}(\beta) d \beta \quad \text { for all } \alpha \in(0, \varepsilon),
$$

as $h^{\prime}(0)=0$. It follows that the set $E=\left\{\alpha \in(0, \varepsilon): h^{\prime \prime}(\alpha) \geq 0\right\}$ has positive measure in each interval $(0, \delta), \delta \in(0, \varepsilon)$.

If inf $A=0$, then $\varphi^{\prime}\left(\alpha_{n}\right)=\psi^{\prime}\left(\alpha_{n}\right)$ for some $\alpha_{n} \downarrow 0$. Then

$$
0=h^{\prime}\left(\alpha_{n}\right)=\int_{0}^{\alpha_{n}} h^{\prime \prime}(\beta) d \beta
$$

and the conclusion follows.

Proof of Theorem 3.4. By Theorem 3.3 it suffices to show that every contractive projection $P: X \rightarrow X$ is semi-band preserving. Let $x, y \in X$ with $y$ simple, and suppose that $P x$ and $y$ are disjoint and $P x \neq 0$. Define functions $\varphi: \mathbb{R} \rightarrow[0, \infty)$ and $\psi: \mathbb{R} \rightarrow[0, \infty)$ by

$$
\varphi(\alpha)=\|P x+\alpha P y\|, \quad \psi(\alpha)=\|P x+\alpha y\| \quad \text { for all } \alpha \in \mathbb{R} .
$$

Obviously, $\varphi$ and $\psi$ are convex and $\varphi(0)=\psi(0)$. As $P$ is a contractive projection, $\varphi(\alpha)=\left\|P^{2} x+\alpha P y\right\| \leq \psi(\alpha)$ for all $\alpha \in \mathbb{R}$. Moreover, $\varphi$ and $\psi$ are both twice continuously differentiable, because $X$ is $D^{2}$-smooth. We can now use the fact that $X$ reflects disjointness and $y$ is simple, to deduce from Definition 3.1(iii) that $\psi^{\prime}(0)=0$ and $\psi^{\prime \prime}(\alpha)$ converges to 0 as $\alpha \rightarrow 0$ along a subset of $[0,1]$ of full measure. Since $\varphi^{\prime \prime}$ is continuous, Lemma 3.5 gives $\varphi^{\prime}(0)=0$ and $\varphi^{\prime \prime}(0)=0$. By using Definition 3.1(ii) we find that $P x$ and $P y$ are disjoint. An arbitrary element $y \in X$ can be approximated by elements $y_{n}$ in $X$ such that the support of each $y_{n}$ is finite and contained in the support of $y$. We conclude that $P$ is semi-band preserving.

The condition on the second derivative of the norm in Definition 3.1 has a natural interpretation in terms of curvature properties of the unit sphere, if the Banach space is finite-dimensional. More precisely, one can show that if $X$ is a $C^{2}$-smooth finite-dimensional Banach space with norm $\varrho$ and the standard basis is 1-unconditional, then $X$ reflecting disjointness is equivalent to saying that for each $x \in S_{X}$ the normal curvature at $x$ in the direction of $y$ is 0 if and only if $x$ and $y$ are disjoint. To prove this one has to note that the normal curvature $k(y)$ at $x$ in the direction $y$, where $y$ is in the tangent space at $x$, is given by $k(y)=$ $N_{x y}^{\prime \prime}(0) /\|\nabla \varrho(x)\|_{2}$. 
4. Contractive projections on $\ell_{p}(X)$. In this section we analyse contractive projections on the vector-valued spaces $\ell_{p}(X)$ and, in particular, the spaces $\ell_{p}\left(\ell_{q}\right)$, where $p$ and $q$ are not equal to 2 . As mentioned in the introduction we distinguish two cases: the unmixed case, where $p, q \in(2, \infty)$ or $p, q \in(1,2)$, and the mixed case, where $p \in(1,2)$ and $q \in(2, \infty)$ or the other way around. We first prove a lemma and subsequently discuss the unmixed case.

Lemma 4.1. Let $X$ be a $C^{2}$-smooth Banach space with norm $\sigma$ such that $D \sigma$ is uniformly bounded on $S_{X}$. Let $p \in(1,2) \cup(2, \infty)$, and let $x, y \in \ell_{p}(X)$. For $\alpha \in \mathbb{R}$ define

$$
\begin{aligned}
N_{k}(\alpha) & :=\sigma(x(k)+\alpha y(k)), \quad k \in \mathbb{N}, \\
\tau(\alpha) & :=\sum_{k=1}^{\infty} N_{k}^{p}(\alpha), \\
N(\alpha) & :=\tau(\alpha)^{1 / p} .
\end{aligned}
$$

Then $\tau$ is $C^{1}, \tau^{\prime}$ is absolutely continuous, $N^{\prime}$ and $N^{\prime \prime}$ exist almost everywhere, and

$$
\begin{aligned}
\text { (1) } \quad \tau^{\prime}(\alpha)= & \sum_{k \in \mathrm{vs}(x) \cap \mathrm{vs}(y)} p N_{k}^{p-1}(\alpha) N_{k}^{\prime}(\alpha)+p \alpha^{p-1} \sum_{k \in \mathrm{vs}(y) \backslash \mathrm{vs}(x)} \sigma^{p}(y(k)), \\
(2) \quad \tau^{\prime \prime}(\alpha)= & \sum_{k \in \mathrm{vs}(x) \cap \mathrm{vs}(y)}\left(p(p-1) N_{k}^{p-2}(\alpha) N_{k}^{\prime}(\alpha)^{2}+p N_{k}^{p-1}(\alpha) N_{k}^{\prime \prime}(\alpha)\right) \\
& +p(p-1) \alpha^{p-2} \sum_{k \in \mathrm{vs}(y) \backslash \mathrm{vs}(x)} \sigma^{p}(y(k)), \\
(3) \quad \tau^{\prime}(\alpha)= & p N^{p-1}(\alpha) N^{\prime}(\alpha), \\
(4) \quad \tau^{\prime \prime}(\alpha)= & p(p-1) N^{p-2}(\alpha) N^{\prime}(\alpha)^{2}+p N^{p-1}(\alpha) N^{\prime \prime}(\alpha)
\end{aligned}
$$

for Lebesgue-almost every $\alpha \in \mathbb{R}$.

Proof. Consider first $k \in \mathbb{N}$ such that $x(k)+\alpha y(k) \neq 0$ for all $\alpha \in \mathbb{R}$. Then $N_{k}$ is a $C^{2}$-function and hence $\left(N_{k}^{p}\right)^{\prime}=p N_{k}^{p-1} N_{k}^{\prime}$ is $C^{1}$. Twice indefinite integration of $\left(N_{k}^{p}\right)^{\prime \prime}$ yields

$$
\begin{aligned}
N_{k}^{p}(\alpha)= & N_{k}^{p}(\beta)+p N_{k}^{p-1}(\beta) N_{k}^{\prime}(\beta)(\alpha-\beta) \\
& +\int_{\beta}^{\alpha} \int_{\beta}^{\alpha}\left(p(p-1) N_{k}^{p-2}(r) N_{k}^{\prime}(r)^{2}+p N_{k}(r)^{p-1}(r) N_{k}^{\prime \prime}(r)\right) d r d s
\end{aligned}
$$

for all $\alpha, \beta \in \mathbb{R}$ with $\alpha \geq \beta$. On the other hand, if $k \in \mathbb{N}$ is such that $x(k)+$ $\gamma y(k)=0$ for some $\gamma \in \mathbb{R}$ then $N_{k}$ is $C^{2}$ on $\mathbb{R} \backslash\{\gamma\}$ and a straightforward computation shows that (5) is true also in this case. 
Next we integrate the right hand side of (2) twice. As $N_{k}$ is convex, we have $N_{k}^{\prime \prime} \geq 0$ almost everywhere, so the right hand side of (2) is a measurable function with values in $[0, \infty]$. Fubini's theorem for positive functions and (5) therefore yield

$$
\begin{gathered}
\int_{\beta}^{\alpha} \int_{\beta}^{s}\left(\sum_{k \in \mathrm{vs}(x) \cap \mathrm{vs}(y)}\left(p(p-1) N_{k}^{p-2}(r) N_{k}^{\prime}(r)^{2}+p N_{k}^{p-1}(r) N_{k}^{\prime \prime}(r)\right)\right. \\
\left.+p(p-1) r^{p-2} \sum_{k \in \mathrm{vs}(y) \backslash \mathrm{vs}(x)} \sigma^{p}(y(k))\right) d r d s \\
=\tau(\alpha)-\tau(\beta)-\left(\sum_{k \in \mathrm{vs}(x) \cap \mathrm{vs}(y)} p N_{k}^{p-1}(\beta) N_{k}^{\prime}(\beta)\right)(\alpha-\beta) \\
-p \beta^{p-1} \sum_{k \in \mathrm{vs}(y) \backslash \mathrm{vs}(x)} \sigma^{p}(y(k))(\alpha-\beta)
\end{gathered}
$$

for all $\alpha \geq \beta$. Since $D \sigma$ is uniformly bounded on $S_{X}$ and $D \sigma(x)=D \sigma(\lambda x)$ for all $\lambda \neq 0$, there exists a constant $C$ such that $\|D \sigma(z)\| \leq C$ for all $z \in X \backslash\{0\}$. Due to Young's inequality we have

$$
\begin{aligned}
\left|p N_{k}^{p-1}(\alpha) N_{k}^{\prime}(\alpha)\right| & \leq p C \sigma(x(k)+\alpha y(k))^{p-1} \sigma(y(k)) \\
& \leq(p-1) C(\sigma(x(k))+|\alpha| \sigma(y(k)))^{p}+\frac{1}{p} C \sigma(y(k))^{p},
\end{aligned}
$$

and it therefore follows from (6) that the right hand side of (2) is an integrable function of $\alpha$ on bounded intervals. From (6), it is clear that $\tau$ is $C^{1}$ and that

$$
\begin{aligned}
\tau^{\prime}(\alpha)= & \sum_{k \in \mathrm{vs}(x) \cap \mathrm{vs}(y)} p N_{k}^{p-1}(\beta) N_{k}^{\prime}(\beta)+p \beta^{p-1} \sum_{k \in \mathrm{vs}(y) \backslash \mathrm{vs}(x)} \sigma^{p}(y(k)) \\
& +\int_{\beta}^{\alpha}\left(\sum_{k \in \mathrm{vs}(x) \cap \mathrm{vs}(y)}\left(p(p-1) N_{k}^{p-2}(r) N_{k}^{\prime}(r)^{2}+p N_{k}^{p-1}(r) N_{k}^{\prime \prime}(r)\right)\right. \\
& \left.+p(p-1) r^{p-2} \sum_{k \in \mathrm{vs}(y) \backslash \mathrm{vs}(x)} \sigma^{p}(y(k))\right) d r
\end{aligned}
$$

for $\alpha \geq \beta$. It follows that $\tau^{\prime}$ is absolutely continuous and that (2) holds. With the aid of Fubini's theorem, it also follows that (1) holds.

Since $\tau(\alpha)=0$ either for all $\alpha$ or for at most one $\alpha \in \mathbb{R}$, we deduce that $N^{\prime}$ and $N^{\prime \prime}$ exist almost everywhere and hence (3) and (4) hold for almost every $\alpha \in \mathbb{R}$.

4.1. The unmixed case. If $X$ reflects disjointness and $p \in(2, \infty)$, then the following assertion is true for $\ell_{p}(X)$. 
Proposition 4.2. If $p \in(2, \infty)$ and $X$ is a $C^{2}$-smooth Banach space with a 1-unconditional basis such that $X$ reflects disjointness and the first and second derivatives of the norm on $X$ are uniformly bounded on $S_{X}$, then $\ell_{p}(X)$ reflects disjointness.

Proof. Let $\sigma$ denote the norm on $X$ and let $x, y \in \ell_{p}(X)$ with $x \notin$ $\operatorname{span}\{y\}$. For each $k \in \mathbb{N}$ we define $N_{k}(\alpha)=\sigma(x(k)+\alpha y(k))$ and $\tau(\alpha)=$ $N^{p}(\alpha)=\sum_{k=1}^{\infty} N_{k}^{p}(\alpha)$. It follows from Theorem 2.2 that both $N$ and $\tau$ are continuously differentiable on $\mathbb{R}$. As $N$ is a convex function, the second derivative $N^{\prime \prime}(\alpha)$ exists almost everywhere and the first condition in Definition 3.1 is satisfied.

By Lemma 4.1, there exists a subset $A$ of $[0,1]$ with Lebesgue measure 1 such that (1)-(4) hold for all $\alpha \in A$. Now assume that $N^{\prime}(0)=0$ and that $N^{\prime \prime}(\alpha)$ converges to 0 as $\alpha \rightarrow 0$ along a subset of $[0,1]$ of full measure. As $N^{\prime}$ is continuous near 0 , it follows that $\tau^{\prime}(0)=0$ and $\tau^{\prime \prime}(\alpha)$ converges to 0 as $\alpha \rightarrow 0$ along a subset of $[0,1]$ of full measure. Since each term in the sums in (2) is nonnegative, we deduce for each $k \in \operatorname{vs}(x) \cap \operatorname{vs}(y)$ that $N_{k}^{\prime \prime}(\alpha)$ converges to 0 as $\alpha \rightarrow 0$ along a subset of $[0,1]$ of full measure and the continuity of $N_{k}^{\prime}$ implies that $N_{k}^{\prime}(0)=0$. As $X$ reflects disjointness, we find that $x(k)$ and $y(k)$ are disjoint in $X$ for all $k \in \operatorname{vs}(x) \cap \operatorname{vs}(y)$. Thus, $x$ and $y$ are disjoint in $\ell_{p}(X)$ and hence the second condition in Definition 3.1 is satisfied.

To prove the third condition, we assume that $x$ and $y$ are disjoint and $y$ is simple. As $y$ is simple, the sums in (1) and (2) consist of finitely many terms. Since $p>2$ and $X$ reflects disjointness, we find that $\tau^{\prime}(0)=0$ and $\tau^{\prime \prime}(\alpha)$ converges to 0 as $\alpha \rightarrow 0$ along a subset of $[0,1]$ of full measure. By subsequently using (3) and (4), we see that $N^{\prime}(0)=0$ and $N^{\prime \prime}(\alpha)$ also converges to 0 as $\alpha \rightarrow 0$ along a subset of $[0,1]$ of full measure, and we are done.

A combination of Proposition 4.2 with Theorems 2.2 and 3.4 immediately gives the following corollary.

Theorem 4.3. If $p \in(2, \infty)$ and $X$ is a $C^{2}$-smooth Banach space with a 1-unconditional basis such that $X$ reflects disjointness and the first and second derivatives of the norm on $X$ are uniformly bounded on $S_{X}$, then every contractive projection on $\ell_{p}(X)$ is an averaging operator and its range admits a block basis.

It is well known that for each $q>2$ the space $\ell_{q}$ is $C^{2}$-smooth and the first and second derivatives of the norm are uniformly bounded on $S_{X}$ (see e.g. [6, Chapter V]). Furthermore, since $\mathbb{R}$ reflects disjointness it follows from Proposition 4.2 that $\ell_{q}$ reflects disjointness if $q>2$. Therefore we have the following result. 
Corollary 4.4. If $p, q \in(2, \infty)$ or $p, q \in(1,2)$, then the range of every contractive projection on $\ell_{p}\left(\ell_{q}\right)$ has a block basis.

Proof. The case $p, q \in(2, \infty)$ is an immediate consequence of Theorem 4.3. For $p, q \in(1,2)$ the assertion follows from the fact that the dual of $\ell_{p}\left(\ell_{q}\right)$ is equal to $\ell_{p^{*}}\left(\ell_{q^{*}}\right)$, where $1 / p+1 / p^{*}=1$ and $1 / q+1 / q^{*}$ $=1$.

4.2. The mixed case. In the mixed case the space $\ell_{p}\left(\ell_{q}\right)$ is not $C^{2}$-smooth and it does not reflect disjointness. Therefore we cannot apply Theorem 3.4 to show that every contractive projection is an averaging operator. Instead of using Theorem 3.4 we show that every contractive projection on $\ell_{p}\left(\ell_{q}\right)$ is semi-band preserving. The argument is quite involved and split up into several steps. We begin by proving the following proposition.

Proposition 4.5. If $p \in(1,2)$ and $X$ is a $C^{2}$-smooth Banach space with a 1-unconditional basis such that $X$ reflects disjointness and the derivative of the norm $\sigma$ on $X$ is uniformly bounded on $S_{X}$, then for all $x, y \in \ell_{p}(X)$ with $x \notin \operatorname{span}\{y\}$ we have:

(a) the function $N(\alpha)=\|x+\alpha y\|$ is continuously differentiable and $N^{\prime \prime}(\alpha)$ exists almost everywhere;

(b) if $\mathrm{vs}(y) \subset \mathrm{vs}(x), N^{\prime}(0)=0$, and $N^{\prime \prime}(\alpha)$ converges to 0 as $\alpha \rightarrow 0$ along a subset of $[0,1]$ of full measure, then $x$ and $y$ are disjoint;

(c) if $x$ and $y$ are disjoint, $y$ is simple, and $\operatorname{vs}(y) \subset \operatorname{vs}(x)$, then $N^{\prime}(0)=0$ and $N^{\prime \prime}(\alpha)$ converges to 0 as $\alpha \rightarrow 0$ along a subset of $[0,1]$ of full measure;

(d) if the second derivative of the norm of $X$ is uniformly bounded on $S_{X}$, $\operatorname{vs}(y) \subset \operatorname{vs}(x)$, and $2 \sigma(y(k))<\sigma(x(k))$ for all $k \in \operatorname{vs}(x)$, then there exists $A \subset[0,1]$ of full measure and $C>0$ such that $N^{\prime \prime}(\alpha) \leq C$ for all $\alpha \in A$;

(e) if $\mathrm{vs}(y) \not \subset \mathrm{vs}(x)$, then $N^{\prime \prime}(\alpha) \rightarrow \infty$ as $\alpha \rightarrow 0$ along a subset of $[0,1]$ of full measure.

Proof. Let $\sigma$ denote the norm on $X$. As in the proof of Proposition 4.2 we define $N_{k}(\alpha)=\sigma(x(k)+\alpha y(k))$ for all $k \in \mathbb{N}$ and $\tau(\alpha)=N^{p}(\alpha)=$ $\sum_{k=1}^{\infty} N_{k}^{p}(\alpha)$. By Theorem 2.2 both $\tau$ and $N$ are continuously differentiable on $\mathbb{R}$. Moreover, $N^{\prime \prime}$ exists almost everywhere, as $N$ is a convex function, and hence part (a) is satisfied.

Next, note that equations (1)-(4) hold for almost every $\alpha \in \mathbb{R}$. Suppose that $\operatorname{vs}(y) \subset \operatorname{vs}(x), N^{\prime}(0)=0$, and $N^{\prime \prime}(\alpha)$ converges to 0 as $\alpha \rightarrow 0$ along a subset of $[0,1]$ of full measure. Clearly the second sums in (1) and (2) are zero in that case. As each term in the first sums of (1) and (2) is nonnegative, we conclude from (3) and (4) that $N_{k}^{\prime}(0)=0$, and $N_{k}^{\prime \prime}(\alpha)$ converges to 0 as $\alpha \rightarrow 0$ along a subset of $[0,1]$ of full measure for each $k \in \operatorname{vs}(x) \cap \operatorname{vs}(y)$. 
Since $X$ reflects disjointness and $\operatorname{vs}(y) \subset \operatorname{vs}(x)$, it follows that $x$ and $y$ are disjoint, which proves part (b).

To prove (c), note that if $x$ and $y$ are disjoint, $y$ is simple and $\operatorname{vs}(y) \subset$ $\operatorname{vs}(x)$, then for each $k \in \operatorname{vs}(y)$ we have $N_{k}^{\prime}(0)=0$ and $N_{k}^{\prime \prime}(\alpha)$ converges to 0 as $\alpha \rightarrow 0$ along a subset of $[0,1]$ of full measure. As $y$ is simple, the sums in (1) and (2) consist of finitely many terms, so that $\tau^{\prime}(0)=0$ and $\tau^{\prime \prime}(\alpha)$ converges to 0 as $\alpha \rightarrow 0$ along a subset of $[0,1]$ of full measure. The assertion now follows from equations (3) and (4).

To prove $(\mathrm{d})$ assume that $\operatorname{vs}(y) \subset \operatorname{vs}(x)$. Then the second sum in (2) vanishes. For each $k \in \operatorname{vs}(x)$ and $\alpha \in[0,1]$ we have

$$
N_{k}(\alpha) \geq \sigma(x(k))-\alpha \sigma(y(k))>(1-\alpha / 2) \sigma(x(k))>\sigma(x(k)) / 2
$$

and

$$
N_{k}^{\prime}(\alpha)=(x(k)+\alpha y(k))^{*} y(k) \leq \sigma(y(k)) \leq \sigma(x(k)) / 2,
$$

where $(x(k)+\alpha y(k))^{*}$ denotes the norming functional of $x(k)+\alpha y(k)$. This implies that $\tau^{\prime}$ is bounded on the set $A$ of those $\alpha \in[0,1]$ for which (1)-(4) hold.

Since $D \sigma$ and $D^{2} \sigma$ are uniformly bounded on $S_{X}=\{x \in X: \sigma(x)=1\}$, there exists a constant $c \in \mathbb{R}$ such that $\|D \sigma(z)\| \leq c$ and $\left\|\sigma(z) D^{2} \sigma(z)\right\| \leq c$ for all $z \in X \backslash\{0\}$. Thus, there exists $C>0$ such that

$$
\begin{aligned}
\mid p(p-1) N_{k}^{p-2}(\alpha) & N_{k}^{\prime}(\alpha)^{2}+p N_{k}^{p-1}(\alpha) N_{k}^{\prime \prime}(\alpha) \mid \\
\leq & p(p-1)\left(\sigma(x(k)+\alpha y(k))^{p-2} c^{2} \sigma(y(k))^{2}\right) \\
& +p(\sigma(x(k)+\alpha y(k)))^{p-2} c \sigma(y(k))^{2} \\
\leq & \frac{p(p-1)}{2} \sigma(x(k))^{p-2} c^{2} \sigma(x(k))^{2}+\frac{p}{2} \sigma(x(k))^{p-2} c \sigma(x(k))^{2} \\
\leq & C \sigma(x(k))^{p},
\end{aligned}
$$

as $p<2$. Therefore $\tau^{\prime \prime}$ is also bounded on $A$. It is now straightforward to deduce from (3) and (4) that $N^{\prime \prime}$ is bounded on $A$.

Finally, to prove (e) we assume that $\operatorname{vs}(y) \not \subset \mathrm{vs}(x)$. In that case the second sum in (2) becomes unbounded as $\alpha \rightarrow 0$. As every term in the first sum of (2) is nonnegative, we conclude that $\tau^{\prime \prime}(\alpha) \rightarrow \infty$ as $\alpha \rightarrow 0$ along a subset of $[0,1]$ on which $N_{k}^{\prime \prime}(\alpha)$ exists for all $k \in \mathrm{vs}(y)$. This subset of $[0,1]$ may be chosen such that it has full measure, as vs $(y)$ is countable. By using (4) we deduce (e).

To prove that a contractive projection on $\ell_{p}(X)$ with $p \in(1,2)$ is semiband preserving, we need to show that $P x$ and $P y$ are disjoint whenever $P x$ and $y$, with $y$ simple, are disjoint. To establish this, it is convenient to write $y=y^{1}+y^{2}+y^{3}$ with each $y^{i}$ disjoint from $P x$ and where $\operatorname{vs}\left(y^{1}\right) \subset \operatorname{vs}(P x)$, $\operatorname{vs}\left(y^{2}\right) \cap \operatorname{vs}(P z)=\emptyset$ for all $z \in \ell_{p}(X)$, and $\operatorname{vs}\left(y^{3}\right) \cap \operatorname{vs}(P x)=\emptyset$, but $\operatorname{vs}\left(y^{3}\right) \subset$ 
$\operatorname{vs}(P z)$ for some $z \in \ell_{p}(X)$. The idea is to prove disjointness of $P y^{i}$ and $P x$ for $i=1,2$ and 3 separately. Let us start by analysing $y^{1}$.

Lemma 4.6. Let $p \in(1,2)$ and $X$ be a $C^{2}$-smooth Banach space with a 1-unconditional basis such that $X$ reflects disjointness and the second derivative of the norm on $X$ is uniformly bounded on $S_{X}$. If $P: \ell_{p}(X) \rightarrow \ell_{p}(X)$ is a contractive projection, then for all $x, y \in \ell_{p}(X)$ with $\operatorname{vs}(y) \subset \operatorname{vs}(P x)$ such that either $y$ is simple or $\sigma(y(k)) \leq \sigma(P x(k))$ for all $k$, we have $\operatorname{vs}(P y) \subset \operatorname{vs}(P x)$.

Proof. Let $x, y \in \ell_{p}(X)$ with $P x \neq 0$. For $\alpha \in \mathbb{R}$ let $\varphi(\alpha)=\|P x+\alpha P y\|$ and $\psi(\alpha)=\|P x+\alpha y\|$. As $P$ is a contractive projection, $\varphi(\alpha) \leq \psi(\alpha)$ for all $\alpha \in \mathbb{R}$ and $\varphi(0)=\psi(0)$. In both cases we can rescale $y$ so that $2 \sigma(y(k))<\sigma(P x(k))$ for all $k \in \operatorname{vs}(P x)$. Since $\operatorname{vs}(y) \subset \operatorname{vs}(P x)$, we know by Proposition 4.5(d) that there exists $C>0$ such that

$$
\left\{\alpha \in[0,1]: \psi^{\prime \prime}(\alpha) \text { exists and } \psi^{\prime \prime}(\alpha) \leq C\right\}
$$

has full measure in $[0,1]$. Hence it follows from Lemma 3.5(c) that $\varphi^{\prime \prime}(\alpha)$ does not go to infinity as $\alpha \rightarrow 0$ along any subset of $[0,1]$ of full measure. By using Proposition 4.5(e) we conclude that vs $(P y) \subset \mathrm{vs}(P x)$.

This lemma has the following consequence.

Lemma 4.7. Let $p \in(1,2)$ and $X$ be a $C^{2}$-smooth Banach space with a 1-unconditional basis such that $X$ reflects disjointness and the second derivative of the norm on $X$ is uniformly bounded on $S_{X}$. If $P: \ell_{p}(X) \rightarrow \ell_{p}(X)$ is a contractive projection and $x, y \in \ell_{p}(X)$ with $y$ simple are such that $y$ and $P x$ are disjoint and $\mathrm{vs}(y) \subset \mathrm{vs}(P x)$, then $P y$ and $P x$ are disjoint.

Proof. For $\alpha \in \mathbb{R}$ define $\varphi(\alpha)=\|P x+\alpha P y\|$ and $\psi(\alpha)=\|P x+\alpha y\|$. As $P$ is a contractive projection, $\varphi(\alpha) \leq \psi(\alpha)$ for all $\alpha \in \mathbb{R}$ and $\varphi(0)=\psi(0)$. We may assume that $P x \neq 0$. Due to Lemma 4.1 and (3), $\varphi$ and $\psi$ are $C^{1}$ and $\varphi^{\prime}$ and $\psi^{\prime}$ are absolutely continuous functions on a neighborhood of 0 . As $y$ and $P x$ are disjoint, $y$ is simple, and $\operatorname{vs}(y) \subset \operatorname{vs}(P x)$, Proposition 4.5(c) shows that $\psi^{\prime}(0)=0$ and $\psi^{\prime \prime}(\alpha) \rightarrow 0$ as $\alpha \rightarrow 0$ along a full subset $A$ of $[0,1]$. By Lemma 3.5, there exists a measurable set $E \subset[0,1]$ such that $E \cap(0, \delta)$ has positive measure for all $\delta>0$ and $0 \leq \varphi^{\prime \prime}(\alpha) \leq \psi^{\prime \prime}(\alpha)$ for all $\alpha \in E$. We may intersect $E$ with the full set $A$ and thus assume that $E \subset A$. Then $\varphi^{\prime \prime}(\alpha) \rightarrow 0$ as $\alpha \rightarrow 0$ along $E$. Applying (2) to $P x$ and $P y$ instead of $x$ and $y$ gives vs $(P y) \subset \operatorname{vs}(P x)$ and for each $k \in \operatorname{vs}(P x) \cap \operatorname{vs}(P y)$ we have

$$
N_{k}^{p-1}(\alpha) N_{k}^{\prime \prime}(\alpha) \rightarrow 0 \quad \text { as } \alpha \rightarrow 0 \text { along } E .
$$

Therefore, for $k \in \operatorname{vs}(P y), N_{k}^{\prime \prime}(\alpha) \rightarrow 0$ along $E$ and hence $N_{k}^{\prime \prime}(\alpha) \rightarrow 0$ as $\alpha \rightarrow 0$ along a full subset of $[0,1]$, since $N_{k}^{\prime \prime}$ is continuous near 0 . Lemma 3.5 further yields $\varphi^{\prime}(0)=0$. From (1) it follows that $0 \leq N_{k}^{p-1}(\alpha) N_{k}^{\prime}(\alpha) \leq \varphi^{\prime}(\alpha)$ for almost every $\alpha$ and each $k \in \operatorname{vs}(P y)$, so that the continuity of $\varphi^{\prime}$ and 
$N_{k}^{\prime}$ gives $N_{k}^{\prime}(0)=0$. Since $X$ reflects disjointness, we deduce that $P x(k)$ and $P y(k)$ are disjoint for all $k \in \mathrm{vs}(P y)$, and hence $P x$ and $P y$ are disjoint.

Lemma 4.7 shows that $P y^{1}$ and $P x$ are disjoint. To prove disjointness for $y^{2}$, we shall use the following lemma.

Lemma 4.8. Let $p \in(1,2)$ and $X$ be a Banach space with a 1-unconditional basis. If $P: \ell_{p}(X) \rightarrow \ell_{p}(X)$ is a contractive projection and $y \in \ell_{p}(X)$, with $\operatorname{vs}(y) \cap \operatorname{vs}(P y)=\emptyset$, then $P y=0$.

Proof. Since vs $(y) \cap \operatorname{vs}(P y)=\emptyset$, we have $\|P y+\alpha y\|^{p}=\|P y\|^{p}+$ $\alpha^{p}\|y\|^{p}$ and $\|P y+\alpha P y\|^{p}=(1+\alpha)^{p}\|P y\|^{p}$ for all $\alpha \in[0,1]$. As $P$ is a contractive projection, we deduce that $(1+\alpha)^{p}\|P y\|^{p} \leq\|P y\|^{p}+\alpha^{p}\|y\|^{p}$, so that

$$
\frac{\|P y\|^{p}}{\|y\|^{p}} \leq \frac{\alpha^{p}}{(1+\alpha)^{p}-1} \quad \text { for all } \alpha \in[0,1] .
$$

Now note that, as $p>1$,

$$
\lim _{\alpha \rightarrow 0} \frac{\alpha^{p}}{(1+\alpha)^{p}-1}=\lim _{\alpha \rightarrow 0} \frac{p \alpha^{p-1}}{p(1+\alpha)^{p-1}}=0
$$

and hence $\|P y\|=0$.

To prove disjointness for $y^{3}$ we need the following result.

Lemma 4.9. Let $p \in(1,2)$ and $X$ be a $C^{2}$-smooth Banach space with a 1-unconditional basis such that $X$ reflects disjointness and the derivative of the norm on $X$ is uniformly bounded on $S_{X}$. If $P: \ell_{p}(X) \rightarrow \ell_{p}(X)$ is a contractive projection and $x, y, z \in \ell_{p}(X)$ with $y$ simple are such that $\operatorname{vs}(y) \subset \operatorname{vs}(P z)$ and $\operatorname{vs}(y) \cap \operatorname{vs}(P x)=\emptyset$, then there exists $z^{\prime} \in \ell_{p}(X)$ such that $\operatorname{vs}(y) \subset \operatorname{vs}\left(P z^{\prime}\right)$ and $\operatorname{vs}\left(P z^{\prime}\right) \cap \operatorname{vs}(P x)=\emptyset$.

Before proving this lemma we give an auxiliary result.

Lemma 4.10. Let $p \in(1,2)$ and $X$ be a $C^{2}$-smooth Banach space with a 1-unconditional basis such that $X$ reflects disjointness and the derivative of the norm on $X$ is uniformly bounded on $S_{X}$. Suppose that $P: \ell_{p}(X) \rightarrow \ell_{p}(X)$ is a contractive projection and define

$$
\Sigma_{P}=\left\{A \subset \mathbb{N}: \operatorname{vs}(P u)=A \text { for some } u \in \ell_{p}(X)\right\} .
$$

Then:

(a) If $\left(A_{i}\right)_{i \in \mathbb{N}} \subset \Sigma_{P}$ and $A_{1} \supset A_{2} \supset \cdots$, then $\bigcap_{i \in \mathbb{N}} A_{i} \in \Sigma_{P}$.

(b) If $A, B \in \Sigma_{P}$ and $a \in A \backslash B$, then there exists $D_{a} \in \Sigma_{P}$ such that $a \in D_{a}$ and $D_{a} \subset A \backslash B$. 
Proof. Let $\left(A_{i}\right)_{i \in \mathbb{N}} \subset \Sigma_{P}$ be such that $A_{i} \supset A_{i+1}$ for all $i \in \mathbb{N}$. By definition there exist $u^{i} \in \ell_{p}(X)$ such that $\operatorname{vs}\left(P u^{i}\right)=A_{i}$ for each $i \in \mathbb{N}$. Put $A=\bigcap_{i \in \mathbb{N}} A_{i}$ and let $w=\left(P u^{1}\right) \chi_{A}$, where $\chi_{A}$ is the indicator function of $A$. Clearly, vs $(w)=A \subset \operatorname{vs}\left(P u^{i}\right)$ for all $i \in \mathbb{N}$. Thus, Lemma 4.6 implies that $\operatorname{vs}(P w) \subset \operatorname{vs}\left(P u^{i}\right)$ for all $i \in \mathbb{N}$ and hence $\operatorname{vs}(P w) \subset A$. Put $B=\operatorname{vs}(P w)$ and remark that $(P w) \chi_{A_{1} \backslash B}=0$. Moreover,

$$
P u^{1}=\left(P u^{1}\right) \chi_{A_{1} \backslash A}+\left(P u^{1}\right) \chi_{A}=\left(P u^{1}\right) \chi_{A_{1} \backslash A}+w,
$$

so that $P u^{1}=P^{2} u^{1}=P\left(\left(P u^{1}\right) \chi_{A_{1} \backslash A}\right)+P w$. Therefore

$$
\left(P u^{1}\right) \chi_{A_{1} \backslash B}=P\left(\left(P u^{1}\right) \chi_{A_{1} \backslash A}\right) \chi_{A_{1} \backslash B}+(P w) \chi_{A_{1} \backslash B}=P\left(\left(P u^{1}\right) \chi_{A_{1} \backslash A}\right) \chi_{A_{1} \backslash B} .
$$

As $P$ is contractive, we find that

$$
\begin{aligned}
\left\|\left(P u^{1}\right) \chi_{A_{1} \backslash B}\right\| & =\left\|P\left(\left(P u^{1}\right) \chi_{A_{1} \backslash A}\right) \chi_{A_{1} \backslash B}\right\| \leq\left\|P\left(\left(P u^{1}\right) \chi_{A_{1} \backslash A}\right)\right\| \\
& \leq\left\|\left(P u^{1}\right) \chi_{A_{1} \backslash A}\right\| .
\end{aligned}
$$

Since vs $\left(P u^{1}\right)=A_{1}$ and $B \subset A$, we conclude that $A=B=\operatorname{vs}(P w)$ and hence $A \in \Sigma_{P}$.

To prove the second assertion let $A, B \in \Sigma_{P}$ and $a \in A \backslash B$. If $A \cap B=\emptyset$, then we can take $D_{a}=A$. So, suppose that $A \cap B=B_{0}$ is not empty. By the first assertion, $B_{0} \in \Sigma$. Now let $u \in \ell_{p}(X)$ be in the range of $P$ and $\operatorname{vs}(u)=A$. It follows from Lemma 4.6 that

$$
\operatorname{vs}\left(P\left(u \chi_{A \backslash B_{0}}\right)\right) \subset A \text { and } \operatorname{vs}\left(P\left(u \chi_{B_{0}}\right)\right) \subset B_{0} .
$$

But also $P\left(u \chi_{A \backslash B_{0}}\right)+P\left(u \chi_{B_{0}}\right)=P u=u$, so that

$$
P\left(u \chi_{A \backslash B_{0}}\right) \chi_{A \backslash B_{0}}=u \chi_{A \backslash B_{0}} .
$$

As

$$
P\left(u_{A \backslash B_{0}}\right)=P\left(u_{A \backslash B_{0}}\right) \chi_{A \backslash B_{0}}+P\left(u_{A \backslash B_{0}}\right) \chi_{B_{0}}
$$

and $\left\|P\left(u \chi_{A \backslash B_{0}}\right)\right\| \leq\left\|u \chi_{A \backslash B_{0}}\right\|$, it follows from (8) that $\left\|P\left(u \chi_{A \backslash B_{0}}\right) \chi_{B_{0}}\right\|=0$. Thus, $P\left(u \chi_{A \backslash B_{0}}\right) \chi_{A \backslash B_{0}}=P u \chi_{A \backslash B_{0}}$ and we can take $D_{a}=A \backslash B_{0}$.

Using this lemma it is now straightforward to prove Lemma 4.9.

Proof of Lemma 4.9. Let $y$ be simple and for each $k \in \operatorname{vs}(y)$, let $D_{k}$ be a set in $\Sigma_{P}$ given in Lemma 4.10(b), where $A=\mathrm{vs}(P z)$ and $B=\mathrm{vs}(P x)$. Put $D=\bigcup_{k \in \operatorname{vs}(y)} D_{k}$ and note that, as vs $(y)$ is finite, there exists $z^{\prime} \in \ell_{p}(X)$ such that $\operatorname{vs}\left(P z^{\prime}\right)=D$, and this completes the proof.

A combination of the lemmas now yields the following theorem.

THEOREM 4.11. If $p \in(1,2)$ and $X$ is a $C^{2}$-smooth Banach space with a 1-unconditional basis such that $X$ reflects disjointness and the second derivative of the norm on $X$ is uniformly bounded on $S_{X}$, then every contractive 
projection on $\ell_{p}(X)$ is an averaging operator and its range admits a block basis.

Proof. Let $x, y \in \ell_{p}(X)$ with $y$ simple be such that $P x$ and $y$ are disjoint. Write $y=y^{1}+y^{2}+y^{3}$ with each $y^{i}$ simple and disjoint from $P x$. Moreover assume that $\operatorname{vs}\left(y^{1}\right) \subset \operatorname{vs}(P x), \operatorname{vs}\left(y^{2}\right) \cap \operatorname{vs}(P w)=\emptyset$ for all $w \in \ell_{p}(X)$, and $\operatorname{vs}\left(y^{3}\right) \subset \operatorname{vs}(P z)$ for some $z \in \ell_{p}(X)$ with $\operatorname{vs}(P z) \cap \operatorname{vs}(P x)=\emptyset$. Then it follows from Lemma 4.7 that $P y^{1}$ and $P x$ are disjoint. Moreover, Lemma 4.8 implies that $P y^{2}=0$ and hence $P y^{2}$ and $P x$ are disjoint. For $y^{3}$ we find by Lemma 4.9 that there exists $z^{\prime} \in \ell_{p}(X)$ such that $\operatorname{vs}\left(y^{3}\right) \subset \operatorname{vs}\left(P z^{\prime}\right)$ and $\operatorname{vs}\left(P z^{\prime}\right) \cap \operatorname{vs}(P x)=\emptyset$. In addition, it follows from Lemma 4.6 that $\operatorname{vs}\left(P y^{3}\right) \subset \operatorname{vs}\left(P z^{\prime}\right)$, so that $P y^{3}$ and $P x$ are disjoint.

Theorem 4.11 has the following consequence for $\ell_{p}\left(\ell_{q}\right)$ spaces.

Corollary 4.12. If $p \in(1,2)$ and $q \in(2, \infty)$, or the other way around, then the range of every contractive projection on $\ell_{p}\left(\ell_{q}\right)$ has a block basis.

Proof. The proof follows from Theorem 4.11, the fact that the dual of $\ell_{p}\left(\ell_{q}\right)$ is equal to $\ell_{p^{*}}\left(\ell_{q^{*}}\right)$, where $1 / p+1 / p^{*}=1$ and $1 / q+1 / q^{*}=1$, and the fact that $\ell_{r}$ reflects disjointness if $r>2$.

5. Conclusions. Combining Corollaries 4.4 and 4.12 with the results from [19, Section 5] yields the following characterization of 1-complemented subspaces of $\ell_{p}\left(\ell_{q}\right)$ spaces.

TheOREM 5.1. If $p, q \in(1, \infty)$ with $p, q \neq 2$, and $Y$ is a subspace of $\ell_{p}\left(\ell_{q}\right)$, then $Y$ is the range of a contractive projection on $\ell_{p}\left(\ell_{q}\right)$ if and only if there exists a basis $\left\{y^{i}\right\}_{i=1}^{\operatorname{dim} Y}$ for $Y$ such that for each $i \neq j$ either $\operatorname{vs}\left(y^{i}\right) \cap$ $\operatorname{vs}\left(y^{j}\right)=\emptyset$ or $\operatorname{vs}\left(y^{i}\right)=\operatorname{vs}\left(y^{j}\right)$ and in that case, $y^{i}(k)$ and $y^{j}(k)$ are disjoint and $\left\|y^{i}(k)\right\|_{q}=\left\|y^{j}(k)\right\|_{q}$ for all $k \in \operatorname{vs}\left(y^{i}\right)$.

Of course, it would be interesting to see if this theorem can be extended to general vector-valued $L_{p}\left(L_{q}\right)$ spaces.

Concerning Theorem 3.4, we remark that if $X$ is a Banach sequence space with norm $\|x\|=\|x\|_{p}+\|x\|_{q}$ and $p, q>2$, then $X$ reflects disjointness and hence every contractive projection on $X$ is an averaging operator and its range has a block basis. However, the theorem cannot be applied if $p=2$ and $q>2$. Nevertheless we believe that the same assertion is true, but, as yet, we cannot prove it. In connection with this problem a general conjecture of Randrianantoanina [20, Conjecture 7.9] is worth mentioning, which asserts that if $X$ is a strictly monotone Banach sequence space with a 1-unconditional basis, and $X$ does not contain an isometric copy of the Euclidean plane, then the range of every contractive projection on $X$ admits a block basis. It is known [19] that this conjecture is true in complex Banach spaces. 


\section{References}

[1] T. Ando, Contractive projections in $L_{p}$ spaces, Pacific J. Math. 17 (1966), 391-405.

[2] S. J. Bernau and H. E. Lacey, The range of a contractive projection on an $L_{p}$-space, Pacific J. Math. 53 (1974), 21-41.

[3] F. Bohnenblust, Subspaces of $\ell_{p, n}$ spaces, Amer. J. Math. 63 (1941), 64-72.

[4] B. Bru, H. Heinich et J.-C. Lootgieter, Itération des applications de dualité dans les espaces d'Orlicz, C. R. Acad. Sci. Paris Sér. I Math. 303 (1986), 745-747.

[5] E. W. Cheney and K. H. Price, Minimal projections, in: Approximation Theory (Lancaster, 1969), Academic Press, London, 1970, 261-289.

[6] R. Deville, G. Godefroy and V. Zizler, Smoothness and Renormings in Banach Spaces, Pitman Monogr. Surveys Pure Appl. Math. 64, Longman Sci. \& Tech., Harlow, 1993.

[7] J. Diestel and J. J. Uhl, Jr., Vector Measures, with a foreword by B. J. Pettis, Math. Surveys 15, Amer. Math. Soc., Providence, RI, 1977.

[8] J. Dieudonné, Foundations of Modern Analysis, Pure Appl. Math. 10, Academic Press, New York, 1960.

[9] R. G. Douglas, Contractive projections on an $L_{1}$-space, Pacific J. Math. 15 (1965), 443-462.

[10] I. Doust, Contractive projections on Lebesgue-Bochner spaces, in: Function Spaces (Edwardsville, IL, 1994), Lecture Notes in Pure and Appl. Math. 172, Dekker, New York, 1995, 101-109.

[11] J. E. Jamison, A. Kamińska and G. Lewicki, One-complemented subspaces of Musielak-Orlicz spaces, J. Approx. Theory 130 (2004), 1-37.

[12] S. Kakutani, Some characterizations of Euclidean space, Japan J. Math. 16 (1939), 93-97.

[13] N. J. Kalton and G. V. Wood, Orthonormal systems in Banach spaces and their applications, Math. Proc. Cambridge Philos. Soc. 79 (1976), 493-510.

[14] A. L. Koldobskiū, Isometries of $L_{p}\left(X ; L_{q}\right)$ and equimeasurability, Indiana Univ. Math. J. 40 (1991), 677-705.

[15] B. Lemmens and O. van Gaans, On one-complemented subspaces of Minkowski spaces with smooth Riesz norms, Rocky Mountain J. Math. 36 (2006), 1937-1955.

[16] I. E. Leonard and K. Sundaresan, Geometry of Lebesgue-Bochner function spacessmoothness, Trans. Amer. Math. Soc. 198 (1974), 229-251.

[17] D. R. Lewis, Ellipsoids defined by Banach ideal norms, Mathematika 26 (1979), $18-29$.

[18] J. Lindenstrauss and L. Tzafriri, Classical Banach Spaces I. Sequence Spaces, Springer, Berlin, 1977.

[19] B. Randrianantoanina, 1-complemented subspaces of spaces with 1-unconditional bases, Canad. J. Math. 49 (1997), 1242-1264.

[20] -, Norm-one projections in Banach spaces, Taiwanese J. Math. 5 (2001), 35-95.

[21] - Contractive projections in Orlicz sequence spaces, Abstr. Appl. Anal. 2004, 133145.

[22] - A disjointness type property of conditional expectation operators, Colloq. Math. 102 (2005), 9-20.

[23] Y. Raynaud, The range of a contractive projection in $L_{p}(H)$, Rev. Mat. Complut. 17 (2004), 485-512.

[24] K. Sundaresan, Smooth Banach spaces, Math. Ann. 173 (1967), 191-199.

[25] K. Sundaresan and S. Swaminathan, Geometry and Nonlinear Analysis in Banach Spaces, Lecture Notes in Math. 1131, Springer, Berlin, 1985. 
[26] L. Tzafriri, Remarks on contractive projections in $L_{p}$-spaces, Israel J. Math. 7 (1969), 9-15.

Mathematics Institute

University of Warwick

CV4 7AL Coventry, United Kingdom

E-mail: lemmens@maths.warwick.ac.uk

Mathematical Insitute

Leiden University

P.O. Box 9512

2300 RA Leiden, The Netherlands

E-mail: vangaans@math.leidenuniv.nl
Department of Mathematics and Statistics

Miami University

Oxford, OH 45056, U.S.A.

E-mail: randrib@muohio.edu

Received November 2, 2005

Revised version December 15, 2006 